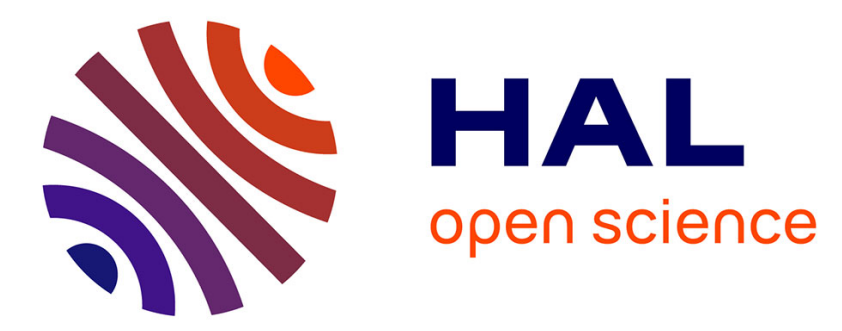

\title{
Transformations métallurgiques accompagnant le réglage du courant critique de microponts de Dayem en film de niobium
}

\author{
Y. Monfort, Daniel Bloyet, J.C. Villegier, D. Pascal
}

\section{- To cite this version:}

Y. Monfort, Daniel Bloyet, J.C. Villegier, D. Pascal. Transformations métallurgiques accompagnant le réglage du courant critique de microponts de Dayem en film de niobium. Revue de Physique Appliquée, 1984, 19 (6), pp.449-454. 10.1051/rphysap:01984001906044900 . jpa-00245216

\section{HAL Id: jpa-00245216 https://hal.science/jpa-00245216}

Submitted on 1 Jan 1984

HAL is a multi-disciplinary open access archive for the deposit and dissemination of scientific research documents, whether they are published or not. The documents may come from teaching and research institutions in France or abroad, or from public or private research centers.
L'archive ouverte pluridisciplinaire HAL, est destinée au dépôt et à la diffusion de documents scientifiques de niveau recherche, publiés ou non, émanant des établissements d'enseignement et de recherche français ou étrangers, des laboratoires publics ou privés. 


\title{
Transformations métallurgiques accompagnant le réglage du courant critique de microponts de Dayem en film de niobium
}

\author{
Y. Monfort \\ LA 251, I.S.M.Ra, Université de Caen, 14032 Caen Cedex, France \\ D. Bloyet
}

G.E.R.S.I.C., I.S.M.Ra, Université de Caen, 14032 Caen Cedex, France

J. C. Villegier

L.E.T.I., C.E.N./G 85 X, 38041 Grenoble Cedex, France

et D. Pascal

Institut d'Electronique Fondamentale, Bâtiment 220, Université Paris XI, 91405 Orsay Cedex, France

(Reçu le 9 décembre 1983, révisé le 7 mars 1984, accepté le 14 mars 1984)

\begin{abstract}
Résumé. - Le courant critique des microponts de Dayem est couramment réglé à sa valeur utile de quelques dizaines de microampères, par application d'impulsions de courant. Nous décrivons dans cet article des observations, en microscopie électronique par transmission, de microponts formés d'une couche mince de niobium microcristallin déposée sur un substrat de silicium. Une croissance cristalline des grains de niobium, accompagnée d'une contamination par le substrat, apparaît au niveau du micropont. Cette contamination semble responsable de la diminution de la température critique du micropont et ainsi de son courant critique à $4,2 \mathrm{~K}$.
\end{abstract}

\begin{abstract}
Metallurgical transformations in $\mathrm{Nb}$ thin film Dayem microbridges related to their critical current adjustment are described : critical current is reduced to a few $10^{-5} \mathrm{~A}$ by application of large current pulses through the microbridge immersed in a liquid $\mathrm{He}$ bath. Transmission electronic microscopy observations show an increase of the mean $\mathrm{Nb}$ grain size close and through the microbridge. $\mathrm{Nb}$ contamination by the silicon substrate is also observed. Critical temperature and critical current reductions of the microbridge are presumably a consequence of this contamination.
\end{abstract}

\section{Introduction.}

Une technique de réglage par impulsions de courant des microponts de Dayem a été proposée par D. Duret et al. [1] en 1975. Cette méthode, améliorée depuis lors [2], est aujourd'hui couramment utilisée. Le phénomène responsable de la réduction contrôlée, souvent très importante, du courant critique n'a jusqu'à présent jamais été identifié. Nous présentons ci-dessous les résultats d'investigations menées par microscopie électronique à transmission permettant de cerner de près ce phénomène dans le cas particulier d'un dépôt de niobium sur un substrat de silicium. Après avoir décrit en 2 la technique de préparation des échantillons, nous présentons au paragraphe 3 l'ensemble de nos observations. Le paragraphe suivant, consacré à l'interprétation des mesures, conduit à l'hypothèse plausible de la contamination du niobium par son substrat et par l'apparition de microcristaux de $\mathrm{Nb}_{5} \mathrm{Si}_{3}$ se plaçant de façon préférentielle au niveau des joints de grains.

\section{Technique de préparation et de réglage du micro- pont.}

La couche de niobium d'épaisseur uniforme d'environ $160 \mathrm{~nm}$ est déposée sur un substrat de silicium monocristallin par évaporation sous ultravide. Le substrat est porté à une température voisine de $400{ }^{\circ} \mathrm{C}$ 
pour obtenir unc tompórature critiquic ot une taille de grain convenables, ainsi qu'une bonne adhérence du dépôt. La photogravure de la couche de niobium suivant une forme de deux carrés se touchant par un sommet définit dans notre application un micropont dont la largeur est voisine de $20 \mu \mathrm{m}$ (Fig. 1). Pour faciliter la prise de contacts électriques, des plots d'or ont préalablement été réalisés sur chaque carré de

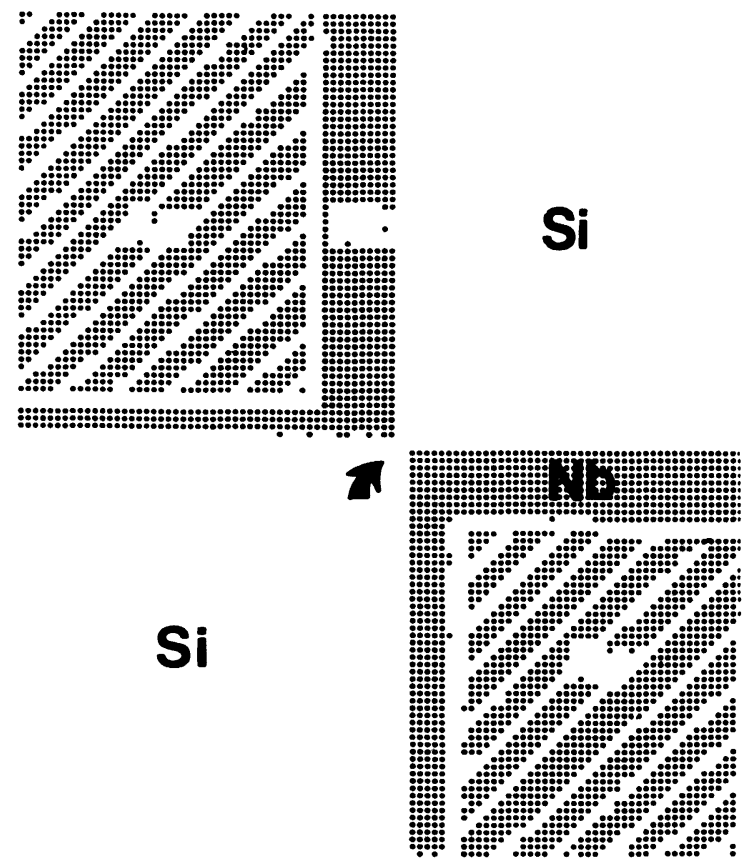

Fig. 1a. - Aspect du micropont de Dayem formé d'une pellicule de niobium déposée sur silicium. Les plots d'or superposés sont utilisés pour les prises de contact électrique. La flèche indique la position du micropont.

[Morphology of the niobium film on silicon Dayem microbridges. Gold pads are placed for electrical contacts purpose. The arrow points at the microbridge.]

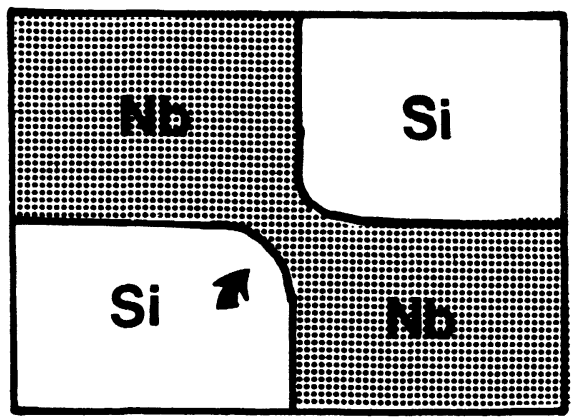

Fig. 1b. - Vue dilatée de la jonction des sommets des carrés de niobium formant le micropont. La largeur de la jonction au niveau de la flèche est d'environ $20 \mu \mathrm{m}$. Au cours du réglage la recristallisation se produit à cet endroit.

[Microbridge enlargement showing the niobium square summits. The arrow points to the recrystallized area by current pulses, this area is about $20 \mu \mathrm{m}$ wide.]

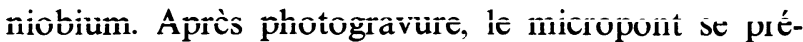
sente comme un supraconducteur de courant critique voisin de $2 \mathrm{~A}$ à $4,2 \mathrm{~K}$. Sa température critique est fonction des conditions de dépôt et varie entre 8 et $9 \mathrm{~K}$.

Pour obtenir l'effet Josephson, il est nécessaire de réduire très fortement ce courant critique. La méthode utilisée est la suivante : à l'échantillon plongé dans l'hélium liquide, on applique une impulsion brève (environ $1 \mu \mathrm{s}$ ) de courant d'amplitude réglable, légèrement supérieure à son courant critique $I_{c_{0}}$. Un dispositif de mesure quatre fils à balayage de courant permet de relever sur oscilloscope la courbe couranttension de l'élément considéré, après chaque impulsion $[1,2]$. Le courant critique $I_{\mathrm{c}}$ peut ainsi être commodément réglé de manière reproductible jusqu'à des valeurs aussi faibles que quelques dizaines de microampères. L'amplitude des impulsions de courant à appliquer pour obtenir une réduction notable de $I_{\mathrm{c}}$ croît très légèrement en fonction de $I_{\mathrm{c}}^{-1}$, sa variation relative est d'environ $7 \%$ pour une variation de $I_{\mathrm{c}_{0}} / I_{\mathrm{c}}$ supérieure à $10^{4}$. Par ailleurs, le cyclage à température ambiante de l'élément réglé a pour effet de réduire encore d'environ $40 \%$ la valeur finale de $I_{c}$, alors que l'application après cyclage d'impulsions d'amplitude judicieusement choisie permet dans certains cas d'aboutir à une recroissance notable de $I_{c}(35 \%)$. Ces réactions marquent l'influence de la métallurgie du micropont sur sa température critique.

\section{Observations en microscopie électronique par trans- mission.}

3.1 PréParation de l'ÉChantillon. - L'observation du micropont nécessite l'élimination locale du substrat de silicium. La méthode de préparation est la suivante : la face supportant le niobium est d'abord protégée à la cire, et une abrasion mécanique du silicium ramène l'épaisseur du substrat à $80 \mu \mathrm{m}$. Un disque de $3 \mathrm{~mm}$ de diamètre, centré sur le micropont, y est découpé pour s'adapter à la dimension requise par le porte-échantillon du microscope. La périphérie de ce disque est protégée à la cire. L'élimination du silicium dans la partie centrale s'effectue par attaque chimique d'un mélange acide nitrique, acide fluorhydrique jusqu'à ce que le silicium apparaisse brun clair par transparence, puis par attaque sélective d'un mélange acide nitrique, acide phosphorique, fluorure d'ammonium, jusqu'à ce que le micropont apparaisse entièrement dégagé du substrat.

3.2 CARACTÉRISATION DU DÉPÔT DE NIOBIUM. - La couche de niobium n'ayant subi aucun traitement électrique est microcristalline. La dimension moyenne des grains est de l'ordre de $20 \mathrm{~nm}$, et leur orientation est aléatoire (Fig. 2). En effet, la diffraction électronique montre des anneaux réguliers correspondant 


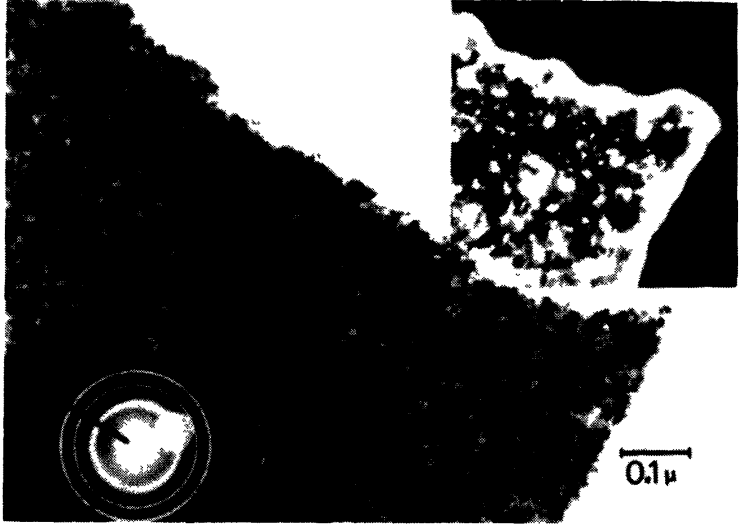

Fig. 2. - Aspect de la pellicule de niobium microcristallisée. L'insertion, en bas à gauche, montre les anneaux de diffraction électronique correspondant aux distances réticulaires successives du niobium. L'insertion, en haut à droite, montre une image de la pellicule, réalisée en champ sombre par sélection d'une portion de l'anneau (110).

[Microcrystallized niobium film aspect. Lower left corner shows electronic diffraction rings corresponding to the successive interreticular spacings of niobium. Upper right corner shows a dark field image of the film corresponding to a portion of the (110) ring.]

à toutes les distances réticulaires successives du niobium (réseau cubique centré, $a=3,30 \AA$ ).

En traitant certains échantillons par une solution aqueuse de soude caustique et d'hypochlorite de sodium qui dissout le niobium en quelques jours, nous avons pu observer l'interface niobium-silicium. Ces observations montrent, outre les réflexions du cristal de silicium, des anneaux (Fig. 3) émanant de microcristaux. Les distances réticulaires $d_{h k l}$ correspondant à ces anneaux coïncident, aux incertitudes de mesure près, à celles de la forme basse température

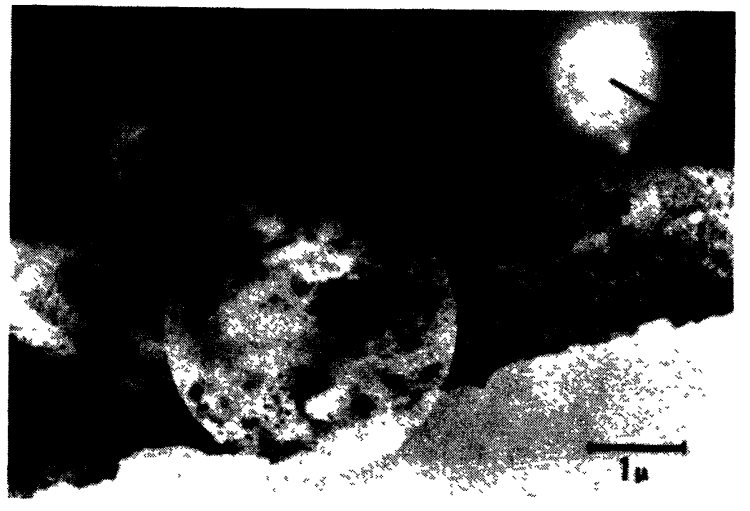

Fig. 3. - Aspect du substrat de silicium aminci. La pellicule de niobium est dissoute. Les points sombres sont les microcristaux de $\mathrm{Nb}_{5} \mathrm{Si}_{3}$. L'encart représente la diffraction électronique de la zone sélectionnée.

[Thinned silicon sustrate. The niobium film is dissolved. $\mathrm{Nb}_{5} \mathrm{Si}_{3}$ microcrystals appear as dark spots. Electron diffraction of the selected area is inserted.] de $\mathrm{Nb}_{5} \mathrm{Si}_{3}$ [3, 4] (Tableau I). Il s'est donc formé pendant la phase de dépôt de niobium un composé microcristallin de $\mathrm{Nb}_{5} \mathrm{Si}_{3}$ à l'interface niobium-silicium.

Tableau I. - Liste des distances réticulaires du composé $\mathrm{Nb}_{5} \mathrm{Si}_{3}$ en forme basse température et comparaison à l'expérience. Réseau quadratique $a=b=6,570 \AA$, $c=11,884 \AA$.

[Reticular spacing file of the $\mathrm{Nb}_{5} \mathrm{Si}_{3}$ low temperature structure type. Computed distances are compared to the measured ones. Tetragonal lattice $a=b=6.570 \AA$, $c=11.884 \AA$.]

\begin{tabular}{|c|c|c|c|}
\hline$h k l$ & $\begin{array}{c}d \text { mesuré } \\
\text { (à } 1,5 \% \text { près) }\end{array}$ & $d$ calculé & $\begin{array}{c}100 . I / I_{\max } \\
\text { mesuré en } \\
\text { diffraction } \mathrm{X}\end{array}$ \\
\hline 211 & $2,83 \AA$ & $2,852 \AA$ & 70 \\
\hline 213 & 2,35 & 2,359 & 100 \\
\hline $\begin{array}{lll}3 & 1 & 0 \\
0 & 0 & 6\end{array}$ & 2,01 & $\begin{array}{l}2,07 \\
1,98\end{array}$ & $\begin{array}{l}70 \\
40\end{array}$ \\
\hline $\begin{array}{ll}02 & 26 \\
134\end{array}$ & 1,71 & $\begin{array}{l}1,69 \\
1,70\end{array}$ & \\
\hline 126 & . 1,66 & 1,64 & \\
\hline $\begin{array}{llll}4 & 1 & 3 \\
2 & 1 & 7,4 & \\
& 2 & 0\end{array}$ & 1,45 & $\begin{array}{l}1,47 \\
1,46\end{array}$ & 50 \\
\hline $\begin{array}{lll}4 & 0 & 4 \\
3 & 1 & 6\end{array}$ & 1,42 & 1,43 & 40 \\
\hline 334 & 1,36 & 1,37 & 50 \\
\hline 228 & 1,27 & 1,25 & 20 \\
\hline 336 & 1,20 & 1,21 & 50 \\
\hline $\begin{array}{lll}523 & \\
4 & 17,440\end{array}$ & 1,15 & $\begin{array}{l}1,166 \\
1,162\end{array}$ & $\begin{array}{l}100 \\
100\end{array}$ \\
\hline
\end{tabular}

3. 3 CARACtÉRISATION DE LA ZONE DU MICROPONT RÉGLÉ. - L'observation des microponts réglés à un courant critique variant de $10 \mu \mathrm{A}$ à $10 \mathrm{~mA}$ révèle une recristallisation locale de la couche de niobium dans la zone de section minimale (Fig. 4). Dans cette zone large de quelques $\mu \mathrm{m}$, la taille des grains (environ $0,2 \mu \mathrm{m}$ ) s'accroît d'un ordre de grandeur par rapport à celle de la couche initiale et devient ainsi voisine de son épaisseur. En ne procédant pas à la seconde étape de l'attaque chimique du silicium mais en dissolvant le niobium recristallisé par la solution aqueuse décrite en 3.2 , nous avons également pu observer l'interface silicium-niobium dans la zone de recristallisation. Celle-ci apparaît amorphe, ce qui conduit à l'interprétation suivante du processus thermique de réglage : les impulsions de courant font approcher, dans les cas limites, le niobium de son point de fusion $\left(T_{\mathrm{f}}=2415^{\circ} \mathrm{C}\right)$, la surface du silicium au contact du niobium entre en fusion $\left(T_{\mathrm{f}}=1410^{\circ} \mathrm{C}\right)$ et la trempe brutale qui suit provoque une solidification sous forme amorphe.

Le réglage de certains microponts a été poussé 


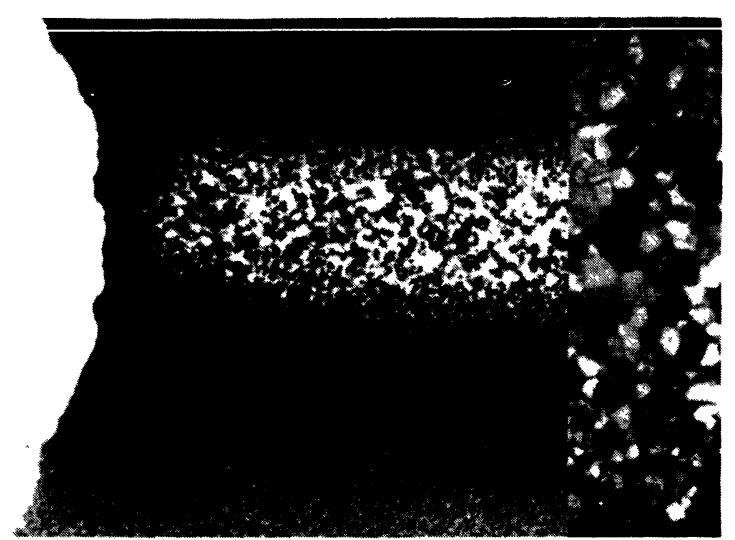

Fig. 4. - Aspect de la zone recristallisée du micropont. La partie droite représente un agrandissement de la zone centrale de la micrographie.

[Microbridge recristallized area view. The right side shows an enlargement of the central part.]

jusqu'à ce que leur courant $I_{\mathrm{c}}$ devienne de l'ordre de quelques microampères, voire nul à $4,2 \mathrm{~K}$. L'aspect de la recristallisation est alors changé (Fig. 5) : il apparaît des cristaux de niobium de plus grande dimension que précédemment, formés autour de perforations de la pellicule de niobium. Nous pensons que l'énergie des impulsions de courant a été suffisamment intense dans ces zones pour entraîner la fusion du niobium et sa recristallisation en plus gros cristaux. Ces derniers contiennent d'ailleurs quelques précipités visibles sur la micrographie mettant en évidence la contamination du niobium.

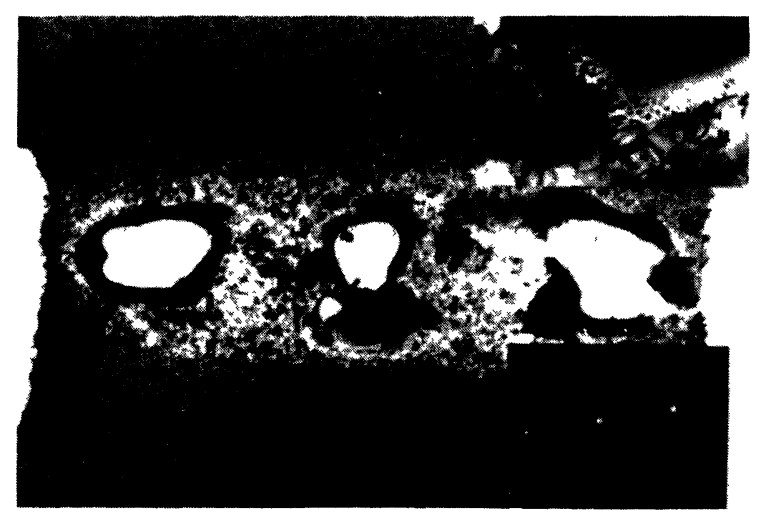

Fig. 5. - Vue d'un micropont présentant des perforations entourées de "gros cristaux" de niobium. L'encart, en haut à droite, montre un agrandissement du cristal situé au centre de la micrographie. L'encart, en bas à droite, représente la diffraction électronique de ce monocristal de niobium $(002,211,21 \overline{1})$.

[Microbridge view showing large niobium crystals around holes. Upper right corner shows an enlargement of the crystal portion located at the micrograph centre. Lower right corner shows the electron diffraction of the niobium monocrystal $(002,211,21 \overline{1})$.]

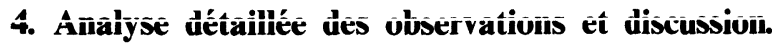

L'examen détaillé du micropont recristallisé montre deux types de défauts :

- Les grains de certains échantillons présentent de petits domaines (Fig. 6) qui ressemblent à ceux que l'on observe lorsque le niobium, vanadium et tantale dissolvent une impureté (oxygène, azote, ...) et deviennent faiblement quadratiques. Néanmoins, ici l'échelle du monocristal est si petite que l'analyse de ce contraste n'a pu être réalisée [5-7],

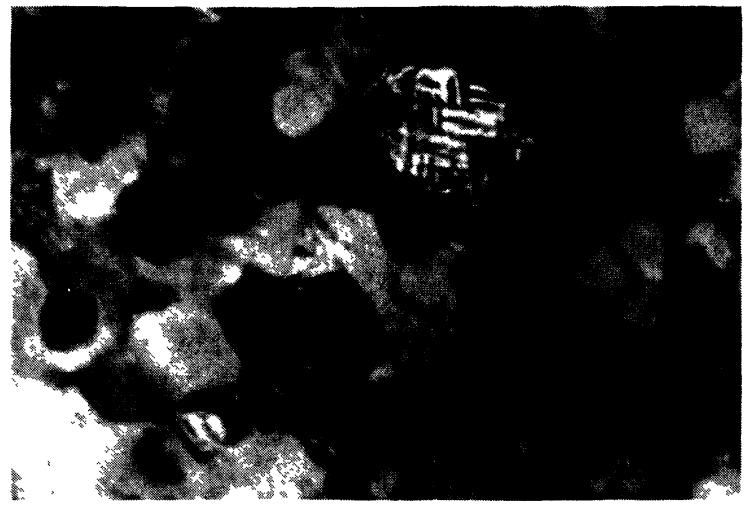

Fig. 6. - Mise en évidence de domaines dans des grains de niobium de la zone recristallisée.

[Domains are revealed in the recrystallized niobium grains.]

- sur d'autres échantillons, c'est la présence de pe $r$ s precipı es qui es p us aci ement mise en evidence. La micrographie en champ sombre de la figure 7 , où ceux-ci sont particulièrement nombreux, montre que certains d'entre eux dessinent les contours des grains de niobium. Il y a dans ce cas concentration de précipités dans les joints de grains.

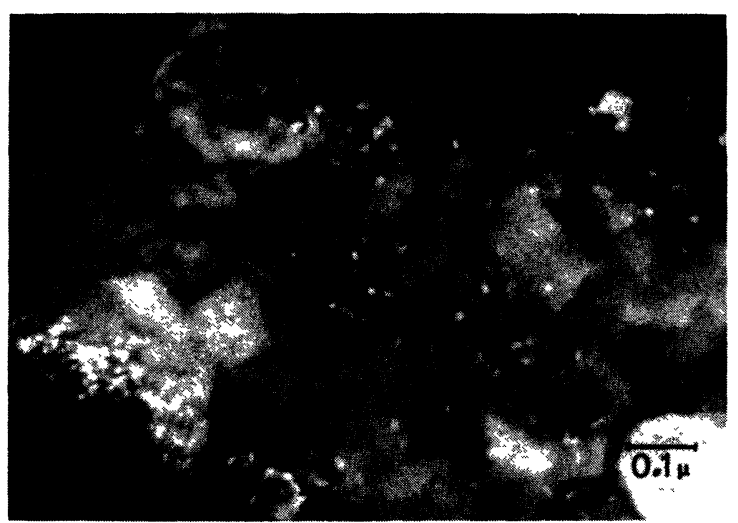

Fig. 7. - Mise en évidence de précipités dans la zone recristallisée. Cette micrographie en champ sombre montre en particulier, au voisinage des lettres $\mathrm{a}, \mathrm{b}$ et $\mathrm{c}$, une répartition de petits précipités entourant des grains de niobium.

[Precipitates are revealed in the recrystallized area. This dark field micrograph shows the distribution of small precipitates around niobium grains. It is particularly clear in the $\mathrm{a}, \mathrm{b}$ and $\mathrm{c}$ points vicinity.] 
Il est peu probable que la contamination du niobium soit due à l'oxygène ou à l'azote. En effet, au moment de la recristallisation, ces gaz ont des pressions partielles négligeables puisque l'échantillon traité est dans l'hélium liquide. Il est par contre très plausible que la contamination provienne du substrat de silicium. Nous avons en effet déjà remarqué en 3.2 la présence de microcristaux de $\mathrm{Nb}_{5} \mathrm{Si}_{3}$ à l'interface niobium-silicium. Pour clarifier ce point, un essai d'analyse du spectre d'énergie de rayons $X$, émis sous le faisceau électronique a été tenté à l'aide d'un système EDAX. Cette expérience révèle une légère augmentation des raies caractéristiques du silicium au niveau de la zone recristallisée. Par ailleurs, l'analyse pour cette zone des distances réticulaires $d_{h k l}$ correspondant aux réflexions autres que celles du niobium (Tableau II) a permis d'identifier la composition des précipités apparaissant en figure 7. Il s'agit, aux incertitudes de mesure près, d'une forme haute température de $\mathrm{Nb}_{5} \mathrm{Si}_{3}[3,4]$. Ces faits expérimentaux démontrent de manière très nette que la diminution du courant critique n'est pas liée à celle de la section du micropont. En effet, le rapport $I_{\mathrm{c}} / I_{\mathrm{c}_{0}}$ a été amené à des valeurs très faibles (couramment $10^{-5}$ ), voire nulle à $4,2 \mathrm{~K}$. De plus, l'étude en température de certains échantillons résistifs à $4,2 \mathrm{~K}$ a permis de retrouver un comportement supraconducteur pour

Tableau II. - Liste des distances réticulaires du composé $\mathrm{Nb}_{5} \mathrm{Si}_{3}$ en forme haute température et comparaison à l'expérience. Réseau quadratique $a=b=10,018 \AA$, $c=5,077 \AA$.

[Reticular spacing file of the $\mathrm{Nb}_{5} \mathrm{Si}_{3}$ high temperature structure type. Computed distances are compared to the measured ones. Tetragonal lattice $a=b=10.018 \AA$, $c=5.077 \AA$.]

\begin{tabular}{|c|c|c|c|}
\hline$h k l$ & $\begin{array}{c}d \text { mesuré } \\
\text { (à } 2 \% \text { près) }\end{array}$ & $d$ calculé & $\begin{array}{c}\text { mesuré en } \\
\text { diffraction } X\end{array}$ \\
\hline & - & - & - \\
\hline 001 & $5,1 \AA$ & $5,07 \AA$ & \\
\hline 310 & 3,09 & 3,16 & 60 \\
\hline 321 & 2,43 & 2,43 & 90 \\
\hline 411 & 2,20 & 2,18 & 100 \\
\hline 222 & 2,04 & 2,09 & 80 \\
\hline 431 & 1,86 & 1,86 & \\
\hline 521 & $\begin{array}{l}1,72 \\
1,70\end{array}$ & 1,74 & 5 \\
\hline 640,513 & 1,36 & 1,38 & 100 \\
\hline
\end{tabular}

une température légèrement inférieure. Enfin, si en limite de réglage, des perforations réduisent effectivement la taille du micropont, elles n'ont pas une section globale suffisante pour leur attribuer la variation correspondante de $I_{\mathrm{c}}$. Par ailleurs, la présence de plusieurs franges d'égale épaisseur dans les cristaux de la zone du recuit indiquent une épaisseur de plusieurs distances d'extinction $(261 \AA$ pour la réflexion $110 \mathrm{de} \mathrm{Nb}$ ) [8]. Cette évaluation indique que l'épaisseur du film de niobium n'a pas fortement diminué.

La seule recristallisation du niobium dans le micropont ne peut expliquer la diminution du courant critique. En effet, si la température critique de dépôts en couches minces microcristallines de certains supraconducteurs devient supérieure à celle d'un monocristal [9], il n'en est pas de même pour le niobium où l'effet inverse se produit pour des tailles moyennes de grains inférieures à environ $10 \mathrm{~nm}$ [10]. La diminution de $I_{c}$ est donc probablement liée à la contamination de la couche de niobium intervenant pendant l'élévation à très haute température du micropont, sous l'effet des impulsions de réglage. L'élément contaminant est ici le silicium du substrat. La présence de la forme haute température de $\mathrm{Nb}_{5} \mathrm{Si}_{3}$, composé pour lequel il n'a pas été observé de propriétés supraconductrices à des températures supérieures à $1 \mathrm{~K}$ [11], témoigne de cette réaction.

\section{Conclusion.}

L'abaissement du courant critique du micropont semble être lié à celui de la température critique du niobium modifié dans cette zone, sous l'effet d'une contamination croissante par le silicium du substrat. Ce travail relatif au substrat de silicium pourra être étendu au support plus utilisé de quartz fondu [1, 2], quand le procédé d'élimination sélective de ce substrat sera mis au point. Pour ce support, la nature de la contamination sera sans doute différent car il existe des réactions d'oxydation du niobium par la silice [12].

\section{Remerciements.}

Nous tenons à remercier vivement $M$. Duret du LETI CENG pour ses conseils avisés et la fourniture des échantillons. 


\section{Bibliographie}

[1] Duret, D., Bernard, P. and Zenatti, D., Rev. Sci. Instrum. 464 (1975) 475.

[2] Pascal, D., Squid 80 (W. de Gruyten, Berlin New York), 1980, p. 417.

[3] Hansen, Constitution of binary alloys (McGraw Hill Book Company) p. 1016.

[4] Powder diffraction file. Inorganic phases. International center for diffraction data (1981) 561.

[5] Monfort, Y., Maisseu, A., Allais, G., Deschanvres, A., Delavignette, P., Phys. Status Solidi (a) 15 (1973) 129.

[6] Nouet, G., Vicens, J., Delavignette, P., Phys. Status Solidi (a) 62 (1980) 449.

[7] Vicens, J., Delavignette, P., Deschanvres, A., J. Microsc. Spectrosc. Electron. 3 (1978) 15.
[8] Hirsh, P. B., Howie, A., Nicholson, R. B., Pashley, D. W., Whelan, M. J., Electron. microscopy of thin crystals (London Butterworths) 1965, p. 100, $212,497$.

[9] Burger, J. P., La supraconductivité des métaux, des alliages et des films minces (Masson et Cie, Editeurs) (1974) 21.

[10] Alessandrini, E. I., Laibowitz, R. B. and Viggiano, J. M., J. Vac. Sci. Technol. 182 (1981) 318.

[11] Matthias, B., Geballe, T. H. and Compton, V. B., Rev. Mod. Phys. 35 (1963) 1.

[12] Miller, G. L., Tantalum and niobium (London Butterworths Scientific Publications) 1959, p. 739. 